\title{
Density presentations of functors
}

\section{B.J. Day}

\begin{abstract}
The article contains the basic theory resulting from the presentation of a dense functor $N: A \rightarrow C$ by means of an expansion $K(k, C) \circ N J k \cong C$, the term dense functor being used instead of the equivalent term left-adequate functor. Results by various authors on the density type of a functor are formulated in the $V$-context for $V$ symmetric monoidal closed and elementary proofs are given. In particular a characterisation theorem containing the well-known results of Beck and Ulmer is established.
\end{abstract}

\section{Introduction}

The concept of density presentation is significant in multilinear algebra as treated from the categorical viewpoint ( $c f$. Day [7] and Day [8]). At the same time it is implicit in providing a uniform treatment of some of the basic characterisation theorems of categorical algebra ( $c f$. Diers [9] and Diers [10]).

By introducing density presentations one attempts to utilise existing analogies between categorical algebra and elementary linear algebra. The precise relationship with elementary analysis is not known because, as yet, there exists no satisfactory method of "measuring" categorical colimits.

The first step is the introduction of density presentations of functors $N: A \rightarrow \mathcal{C}$ as expansions $K(k, C) \circ N J K \cong C$ indexed by $k \in K$. In Section 2 the relationship of presentations to Kan extensions is discussed. The basic characterisation theorem is established in Section 3.

Received 2 February 1977. The author gratefully acknowledges the support of a Postdoctoral Fellowship from the Australian Research Grants Committee. 
The remainder of the article is devoted to examples and Section 5 deals with the important example of linear monads.

Because one aim of the article is to describe density presentations in the general context of $V$-category theory we shall assume that, unless otherwise stated, the categorical algebra is relative to a fixed and suitably complete symetric monoidal closed ground category $V=(V, \otimes, I, \ldots)$. We mainly adhere to the notation and terminology of Eilenberg and Kelly [11] and Mac Lane [21].

We note that the term dense functor (UImer [22]) is used here instead of the term left-adequate functor (Isbell [16]) without intending to justify one or discard the other. The closely related (but much stronger) condition which reduces to topological density when $V=R^{+}$(the positive real numbers as described by Lawvere [18]) is referred to here as Cauchy density.

\section{Density presentations}

Given a category $C$ we denote the discrete category associated with $C$ by $|C|$. A presentation of a functor $N: A \rightarrow C$ consists of an index functor $J: K+A$, a coefficient functor $K: K^{\circ \mathrm{p}} \otimes|\mathrm{C}| \rightarrow V$, and a structure transformation $K(k, C) \rightarrow C(N J k, C)$ which is natural in $k \in K$ and $C \in|C|$. In practice the presentation may be "partially natural" in $C$.

If $S: K^{\mathrm{OP}} \rightarrow V$ and $T: K \rightarrow C$ are functors then we denote their "mean" tensor product (in the sense of Borceux and Kelly [5]) by $S k \circ T K$. Thus, when it exists, $S k \circ T k$ has the defining property

$$
\mathcal{C}(S k \circ T k, C) \cong \int_{k}[S k, \mathcal{C}(T k, C)]
$$

naturally in $C \in \mathcal{C}$.

A presentation is called strong if the tensor product $K(k, C) \circ \mathrm{NJK}$ exists in $C$ and the resultant transformation

$$
\xi_{C}: K(k, C) \circ N J k \rightarrow C
$$

is a strong epimorphism in $\mathcal{C}$. 
A presentation is called a density presentation if the tensor product $K(k, C) \circ N J k$ exists in $C$ and the resultant transformation

$$
\xi_{C}: K(k, C) \circ N J k \rightarrow C
$$

is a regular epimorphism in $C$ such that $C(N A, \xi)$ is an epimorphism for all $A \in A, C \in C$, and $\bar{\xi}: K(k, C) \circ A(A, H k) \rightarrow C(N A, K(k, C) \circ N J k)$ is an epimorphism for all $A \in A, C \in \mathcal{C}$. Thus, if $N$ is full and

faithful then the conditions for a density presentation state that the objects $N A$ are required to be "projective" with respect to the colimits involved in the presentation.

PROPOSITION 1.1. (a) If $(J, K, \xi)$ is a strong presentation of $N: \mathrm{A} \rightarrow \mathrm{C}$ then $N$ strongly generates $\mathrm{C}$.

(b) If $(J, K, \xi)$ is a density presentation of $N: A \rightarrow C$ then $N$ is dense.

Proof. (a) The following diagram commutes:

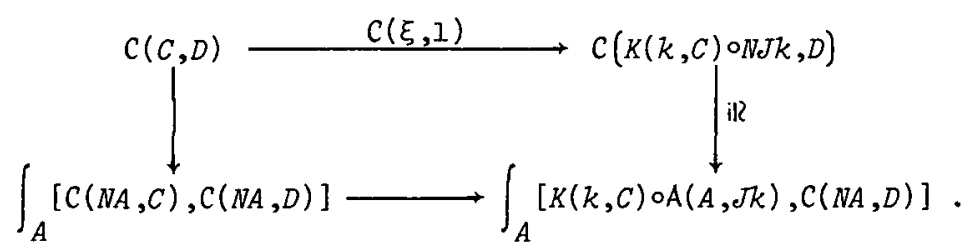

Thus the transformation $\mathcal{C}(C, D) \rightarrow \int_{A}[\mathcal{C}(N A, C), \mathcal{C}(N A, D)]$ is a strong monomorphism because $\mathcal{C}(\xi, 1)$ is a strong monomorphism.

(b) The proof is similar to that for (a). Let

$$
E \stackrel{\rightarrow}{\rightarrow}(k, C) \circ N J k \stackrel{\xi}{\longrightarrow} C
$$

be a coequaliser diagram for $\xi_{C}$ and consider the resultant coequaliser diagram

$$
K(k, E) \circ N J k \rightarrow E \stackrel{\rightarrow}{\rightarrow} K(k, C) \circ N J k \rightarrow C
$$

By naturality we obtain a factorisation 


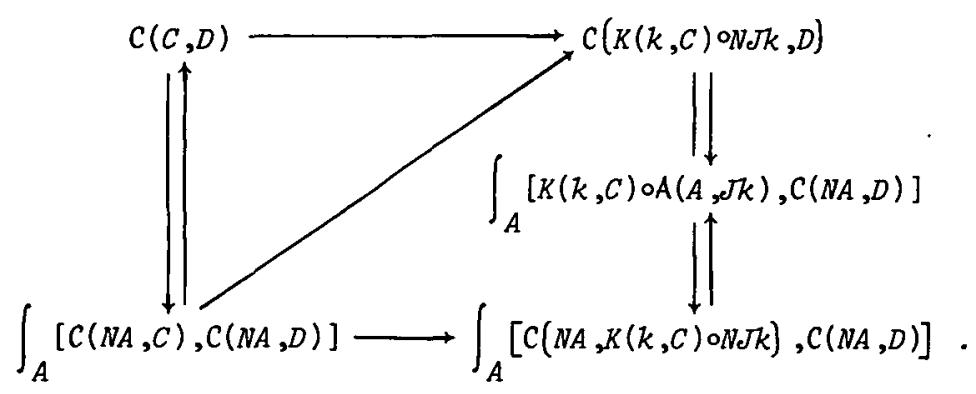

The transformation $\mathcal{C}(C, D) \rightarrow \int_{A}[\mathcal{C}(N A, C), \mathcal{C}(N A, D)]$ is a monomorphism by (a) and is a retraction because the lower arrow is a monomorphism since $C(N A, \xi)$ is an epimorphism. Thus the transformation is an isomorphism, as required. $/ /$

A density presentation is called a strict presentation if both $\xi$ and $\vec{\xi}$ are isomorphisms. Indeed, more complex density presentations than those introduced here do arise but we shall primarily be concerned with strict presentations.

PROPOSITION 1.2 (The Representation Theorem). If $(J, K, \xi)$ is a strict presentation of $N: A+C$ then the induced transformation

$$
\int_{A}[C(N A, C), G A] \rightarrow \int_{k}[K(k, C), G J k]
$$

is an isomorphism for all $N: A^{\text {OP }} \rightarrow V$ and $C \in \mathcal{C}$.

Proof. We have

$$
\begin{aligned}
\int_{A}[C(N A, C), G A] & \cong \int_{A}[\mathrm{C}(N A, K(k, C) \circ N J k), G A] \\
& \cong \int_{A}[K(k, C) \circ A(A, J k), G A] \\
& \cong \int_{k}\left[K(k, C), \int_{A}[A(A, J k), G A]\right] \\
& \cong \int_{k}[K(k, C), G J k]
\end{aligned}
$$

on interchanging limits

by the standard representation theorem.

PROPOSITION 1.3. If $(J, K, \xi)$ are such that 


$$
K(k, C) \circ \mathrm{A}(A, J k) \cong \mathrm{C}(N A, C)
$$

for all $A \in A$ and $C \in C$ then $\xi: K(k, C) \circ N J k \rightarrow C$ is an isomorphism iff $N$ strongly generates $C$ and $K(k, C) \circ A(A, J k) \cong C(N A, K(k, C) \circ N J k)$ for alz $A \in A$ and $C \in C$.

The proof is straightforward.

Associated with a presentation $P=(J, K, \xi)$ of $N: A \rightarrow C$ for which

$$
K(k, C) \circ A(A, J k) \cong C(N A, C)
$$

for all $A \in A$ and $C \in C$, is the category $P C \subset C$ of $P$-objects defined by $P C=\left\{C \in C ; C(\dot{C}, D) \cong \int_{k}[K(k, C), C(N J k, D)]\right.$ for all $\left.D \in C\right\}$. If $N A \in P C$ for all $A \in A$ then $N: A \rightarrow P C$ is dense because

$$
\begin{aligned}
\int_{A}[\mathrm{C}(N A, C), \mathrm{C}(N A, D)] & \cong \int_{A}[K(k, C) \circ \mathrm{A}(A, J k), \mathrm{C}(N A, D)] \\
& \cong \int_{k}[K(k, C), \mathrm{C}(N J k, D)] \\
& \cong \mathrm{C}(C, D) .
\end{aligned}
$$

In particular, given a strict presentation $(J, K, \xi)$ of $N$, each full subcategory of A through which $J$ factors has such a "closure" in $C$.

\section{Kan extensions and adjoints}

PROPOSITION 2.1. Let $(J, K, \xi)$ be a strict presentation of $N: A \rightarrow C$. Then $F: A \rightarrow B$ has a left Kan extension along $N$ if $K(k, C) \circ F J k$ exists in $B$ for all $C \in C$.

Proof.

$$
\begin{aligned}
K(k, C) \circ F J k & \cong\left(\int^{k} K(k, C) \otimes A(A, J k)\right) \circ F A \text { by the representation theorem } \\
& \cong C(N A, C) \circ F A . \quad / /
\end{aligned}
$$

PROPOSITION 2.2. Let $(J, K, \xi)$ be a strict presentation of $N: A \rightarrow C$. Then $F: C \rightarrow B$ is a left Kan extension along $N$ of its restriction by $N$ iff $F$ preserves $K(k, C)$ o NJk for all $C \in \mathcal{C}$.

Proof. $\quad F C \cong F(K(k, C) \circ N J k)$ so we have 
$F C \cong K(k, C) \circ F N J k$

$$
\begin{aligned}
& \cong\left(\int^{k} K(k, C) \otimes A(A, J k)\right) \circ F A \text { by the representation theorem } \\
& \cong C(N A, C) \circ F N A
\end{aligned}
$$

and the result follows. //

Let $N: A \rightarrow C$ be a functor and let $N^{\prime}: C \rightarrow\left[A^{\circ p}, V\right]$ be the induced Yoneda functor.

PROPOSITION 2.3. Let $N: A \rightarrow C$ be dense by strict presentation $(J, K, \xi)$.

(a) If $N$ is fully faithful then $F: C \rightarrow B$ has a right $N^{\prime}$-adjoint iff $F(K(k, C) \circ N J k) \cong K(k, C)$ o FNJk for alz $C \in \mathcal{C}$.

(b) $F: C \rightarrow B$ has a right adjoint iff it commites with $K(k, C) \circ N J k$ for all $C \in \mathcal{C}$ and $F N$ has a right N-adjoint.

(c) If $F \underset{N}{R}: B \rightarrow C$ then $R$ has a left adjoint $\bar{F} C=K(k, C) \circ$ FJk if this colimit exists in $B$.

Proof. (a) Suppose $F \overparen{N^{\top}} R$. Then

$$
\begin{aligned}
B(F C, B) & \cong\left[A^{\circ \mathrm{p}}, V\right]\left(N^{\prime} C, R B\right) \\
& \cong\left[A^{\mathrm{op}}, V\right]\left(N^{\prime} C, B(F N-, B)\right],
\end{aligned}
$$

because

$$
B(F N A, B) \cong\left[A^{\circ \mathrm{p}}, V\right]\left(N^{\prime}(N A), R B\right) \cong R B(A)
$$

by the representation theorem and the full faithfulness of $N$. Thus

$$
\begin{aligned}
B(F C, B) & =\int_{A}[C(N A, C), B(F N A, B)] \\
& \cong B(C(N A, C) \circ F N A, B) \text { for all } C \in C \text { and } B \in B .
\end{aligned}
$$

Thus $F C \cong C(N A, C) \circ F N A$ for all $C \in C$ and Proposition 2.2 applies. Conversely, if $F(K(k, C)$ oNJk $) \cong K(k, C) \circ$ FNJk for all $C \in \mathcal{C}$ then we define $R$ by $R(B)(A)=B(F N A, B)$ and Proposition 2.2 applies.

(b) Suppose $F N \underset{N}{\longrightarrow} R$ and $F(K(k, C) \circ N J k) \cong K(k, C) \circ F N J k$. By Proposition 2.2, $F$ is the Kan extension of its restriction by $N$. Thus $F C \cong \mathcal{C}(N A, C) \circ F N A$ whence $F \dashv R$ because 


$$
\begin{aligned}
B(F C, B) & \cong B(C(N A, C) \circ F N A, B) \\
& \cong \int_{A}[C(N A, C), B(F N A, B)] \\
& \cong \int_{A}[C(N A, C), C(N A, R B)] \\
& \cong C(C, R B) \text { because } N \text { is dense. }
\end{aligned}
$$

Conversely, if $F \rightarrow R$ then $F$ preserves colimits and

$$
B(F N A, B)=\mathcal{C}(N A, R B),
$$

as required.

(c)

$$
\begin{aligned}
B(\bar{F} C, B) & =B(K(k, C) \circ F J k, B) \\
& \cong \int_{k}[K(k, C), B(F J k, B)] \\
& \cong \int_{k}[K(k, C), C(N J k, R B)] . \\
& \cong C(K(k, C) \circ N J k, R B) \\
& \cong C(C, R B) . \quad / /
\end{aligned}
$$

3. The characterisation theorem

We suppose that $N: A \rightarrow C$ is dense by a strict presentation $P=(J, K, \xi)$.

THEOREM 3.1. Suppose $F \underset{N}{\longrightarrow} R: B \rightarrow C$ and $\bar{F} C=K(k, C) \circ F J k$ exists in $B$ for all $C \in \mathcal{C}$. Then

(a) $\vec{F} \rightarrow R$ is a reflective embedding if $R$ reflects isomorphisms and preserves $K(K, R B) \circ F J k$ for all $B \in B$ and $N \cong R F$ : in this case $F$ is dense;

(b) $\bar{F} \dashv R$ is an equivalence iff $R$ reflects isomorphisms and preserves $K(k, C) \circ F J k$ for all $C \in \mathcal{C}$ and $N \cong R F$;

(c) $\bar{F} \rightarrow R$ is an isomorphism iff the conditions of (b) hold and $R$ creates isomorphisms.

Proof. (a) Applying $R$ to the counit of $\bar{F} \rightarrow R$ we obtain: 


$$
\begin{aligned}
\overline{R \bar{F} R B} & =R(K(k, R B) \circ F J k) \\
& \cong K(k, R B) \circ R F J k \\
& \cong K(k, R B) \circ N J k \\
& \cong R B,
\end{aligned}
$$

so $\bar{F} R \cong I$, as required. Thus $F$ is dense by index $J$ and coefficients $K(k, R B)$.

(b) Necessity of the conditions is clear. Conversely, by (a), it remains to show that the unit of $\bar{F} \dashv R$ is an isomorphism. We have

$$
\begin{aligned}
C & \cong K(k, C) \circ N J k \\
& \cong K(k, C) \circ R F J k \\
& \cong R(K(k, C) \circ F J k) \\
& \cong R \bar{F} C
\end{aligned}
$$

as required.

(c) This follows from (b).

DEFINITION 3.2. An object $D \in B$ is called P-presentable if $B(D,-)$ preserves $K(k, C)$ o $F J k$ for all $C \in B$.

THEOREM 3.3. Suppose $N$ is fully faithful, $F \underset{N}{\longrightarrow} R: B \rightarrow C$, and $\vec{F} C=K(k, C) \circ F J k$ exists in $B$ for all $C \in C$. Then $\vec{F} \rightarrow R$ is an equivalence iff $N \cong R F, F$ is fully faithful and strongly generating, and each object FJk, $k \in K$, is P-presentable.

Proof. Necessity of the conditions is clear. For sufficiency first note that $R$ reflects isomorphisms because $R f$ an isomorphism implies $\mathcal{C}(N A, R f) \cong B(F A, f)$ is isomorphism for all $A \in A$, implies $f$ an isomorphism since $F$ strongly generates $B$. By Theorem 3.1 it remains to prove that $R(K(k, C) \circ F J k) \cong K(k, C) \circ N J k \cong C$ for all $C \in C$. Because NJ strongly generates $C$ this follows from

$$
\begin{aligned}
C(N J h, R(K(k, C) \circ F J k)) & \cong B(F J h, K(k, C) \circ F J k) \\
& \cong K(k, C) \circ B(F J h, F J k) \\
& \cong K(k, C) \circ A(J h, J k) \\
& \cong C(N J h, C) \text { for all } h \in K . /
\end{aligned}
$$

\section{Examples}

EXAMPLE 4.1. If $N: A \rightarrow C$ is an arbitrary dense functor then there 
exists a standard coend presentation of $N$ with $K=A, J=1: A \rightarrow A$, $K(A, C)=\mathcal{C}(N A, C)$, and

$$
\xi: C(N A, C) \circ N A \cong C .
$$

It is a simple matter to recover the following from Theorem 3.3.

\section{THEOREM 4.1.1 (Bunge [6]). A category $B$ is equivalent to} $\left[D^{\circ}, V\right]$ for some small $D$ iff $B$ is cocomplete and has a small strongly generating subcategory $D$ such that, for each $D \in D, B(D,-): B \rightarrow V$ preserves all colimits. //

EXAMPLE 4.2. If $N: A \rightarrow C$ is dense then there exists a commacategory density presentation of $N$. If $P$ and $Q$ denote the projections

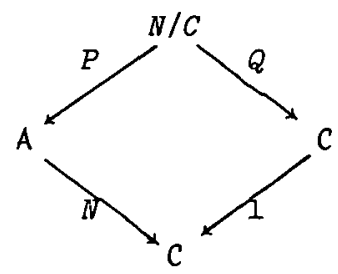

then we obtain a natural isomorphism

$$
\int_{A} B(F N A, G A) \cong \int_{k} B(F Q k, G P k)
$$

which holds for all $F: C^{\mathrm{OP}} \rightarrow B$ and $G: A^{\mathrm{OP}} \rightarrow B$. Thus we obtain

$$
C(Q k, C) \circ N P k \cong C \text {. }
$$

Hence we recall that the $V$-comma category $N / M$ of functors $N: A \rightarrow C+B: M$ is constructed by taking as objects the morphisms $f \in C_{0}(N A, M B)$ and defining the objects of morphisms by means of pull-back diagrams of the form

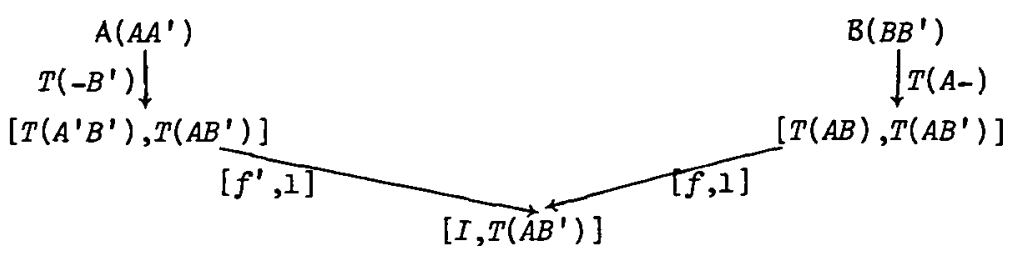

where $T(A B)=C(N A, M B)$. 
EXAMPLE 4.3. Let $T=(T, \mu, \eta)$ be a monad on $C$ with Kleisli category $A=C_{T}$ and Eilenberg-Moore category $C^{\top}$. Let $N: A \rightarrow C^{\top}$ be the comparison functor and let $D$ be the free $V$-category on the category $\{2 \underset{s}{\stackrel{m}{\rightleftarrows}} 1\}$.

Let $K=D \otimes C^{\top}$ with index functor $J: K \rightarrow A$ defined by

$$
\begin{aligned}
& J(m,(C, \zeta))=\mu_{C}: T^{2} C+T C, \\
& J(s,(C, \zeta))=T \zeta: T^{2} C+T C,
\end{aligned}
$$

for each T-algebra $(C, \zeta)$. The coefficient functor $K: K^{o p} \otimes C^{\top} \rightarrow V$ is then defined by

$$
\begin{aligned}
K(d,(C, \zeta),(D, \zeta)) & =I \otimes C^{\top}(C, D) \\
& \cong C^{\top}(C, D) .
\end{aligned}
$$

Then we have

$$
\begin{aligned}
K(d, D, C) \circ N J(d, D) & =c^{\top}(C, D) \circ N J(d, D) \\
& \cong \int^{d} N J(d, C) \\
& \quad \text { by the representation theorem applied to } D \in C^{\top} \\
& \cong \operatorname{colim} N J(d, C) \\
& \cong C
\end{aligned}
$$

because $T^{2} C \underset{T \zeta}{\stackrel{\mu}{\longrightarrow}} T C \stackrel{\zeta}{\longrightarrow} C$ is a coequaliser diagram in $C^{T}$. This

isomorphism is natrual in $C \in C^{\top}$ and the coequaliser diagram is preserved by each functor $C^{\top}(N A,-): C^{\top} \rightarrow V$; hence we obtain a density presentation of $N$. From Theorem 3.1 we obtain:

THEOREM 4.4.1 (Beck). An adjunction $F \dashv U: B \rightarrow C$ is monadic iff $U$ creates coequalisers of U-split pairs. //

On applying the underlying-set functor $V: U \rightarrow S$ to Proposition 1.2 , we obtain: 
PROPOSITION 4.3.2. The comparison functor $N: \mathrm{C}_{T} \rightarrow \mathrm{C}^{\top}$ is dense and, for each algebra $(C, \zeta) \in C^{\top}$, the natural transformations from $C^{\top}(N-, C)$ to a prealgebra $G: A^{O P} \rightarrow V$ correspond to the elements in the equaliser of

$$
V G C \underset{V G T \zeta}{\stackrel{V G \mu}{\longrightarrow}} V G T C
$$

where $\mu$ and $T \zeta$ are regarded as morphisms in $\mathcal{C}_{T} . \quad / 1$

More generally we have:

PROPOSITION 4.3.3. If $F \rightarrow U: B \rightarrow C$ and $\varepsilon: F U \rightarrow I$ is a regular epimorphism then the full image of $F$ is dense in $B$ if $B$ has kernel pairs.

Proof. This is essentially the same as for the monad case except that one presents each $B \in B$ as a coequaliser of the form

$$
D(B) \stackrel{\alpha}{\stackrel{\alpha}{\longrightarrow}} F U B \stackrel{\varepsilon}{\longrightarrow} B
$$

where $D$ is the functorial kernel pair of $\varepsilon$. //

This example admits an evident generalisation. One may suppose that there is a given a functor $N: A \rightarrow C$, a "diagram" $D$, and functors $J: D \otimes C \rightarrow A$ and $H: D^{O P} \rightarrow V$ together with a natural transformation $\alpha_{d C}: H d \rightarrow C(N J(d, C), C)$ such that

$$
\int^{D} H(d) \cdot N J(d, c) \cong C
$$

holds and this coend is preserved by each of the functors $C(N A,-)$, $A \in A$. Then one obtains a strict presentation of $N$ on taking the coefficient functor to be

$$
K: D^{\mathrm{op}} \otimes \mathrm{C}^{\mathrm{op}} \otimes C \stackrel{H \otimes \mathrm{homc}}{\longrightarrow} V \otimes V \stackrel{\otimes}{\longrightarrow} v .
$$

EXAMPLE 4.4. Let $V=S$ and let $C$ be a category with canonical $E$ - $M$ factorisations for a proper $E-M$ factorisation system on $C$ (Freyd and Kelly [12]). Let $M$ also denote the category whose objects are those of $C$ and whose morphisms are those in $M$. Let $A$ be a full 
subcategory of $C$ which is closed under E-images and let $N: A \rightarrow C$ denote the inclusion (usually omitted). On taking $K=A \cap M$ and $K(A, C)=M(A, C)$ we have

$$
\int^{A} M(A, C) \times \mathcal{C}(B, A) \cong \mathcal{C}(B, C)
$$

naturally in $C \in M$ and $B \in A$. Thus, if each object $C \in \mathcal{C}$ is the union $\int^{A} M(A, C) \cdot A$ of its subobjects which lie in $K$ then $N: A \rightarrow C$ is dense and

$$
\int_{A}[C(A, C), G A] \cong \int_{M}[M(A, C), G A]
$$

for each functor $G: A^{o p} \rightarrow S$. It follows from Proposition 1.3 that $\int^{A} M(A, C) \cdot A \cong C$ if $A$ strongly generates $C$ and each $A \in A$ is $P$-presentable for this $P$. In the case $C=S$ any category $A \subset S$ which is closed under surjective images in $S$ satisfies these conditions.

This presentation occurs in the study of pro-objects and modelinduced monads (see Appelgate and Tierney [1], Hofmann [15], Kennison and Gildenhuys [17], and Lim [19]). Given $A \subset C$ we form

$$
\begin{aligned}
& P C=\left\{C \in C ; \int^{A} M(A, C) \cdot A \cong C\right\} \\
& =\{C \in C ; \underset{M(A, C)}{\operatorname{colim}} A \cong C\} .
\end{aligned}
$$

PROPOSITION 4.4.1. If $P C$ is closed in $C$ under E-quotients and colim $A \in P C$ for all $C \in C$ then $P C$ is coreflective in $C$. $M(A, C)$

Proof. The coreflection $R C$ of $C \in C$ is defined by the $E-M$ factorisation of the canonical transformation colim $A \rightarrow C$. //

We generalise a result of Lim [19], Theorem 5.1.

THEOREM 4.4.2. If there exists $U \dashv G: S \rightarrow P C$ then $U G$ generates the density comonad on $U_{0}=U N$. 
Proof.

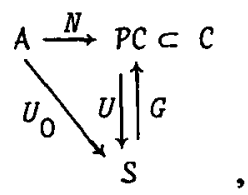

$$
\begin{aligned}
G X & \cong \int^{A} P C(A, G X) \cdot A \text { by the representation theorem } \\
& \cong \int^{A} S(U A, X) \cdot A
\end{aligned}
$$

whence $\quad U G X \cong \int^{A} S\left(U_{0} A, X\right) \cdot U_{0} A \cdot \quad / /$

COROLLARY 4.4.3. If $P C$ is comonadic with respect to $G=U G$ then $P C$ is category equivalent to the category of coalgebras model-induced by $U_{0}=U N . \quad / 1$

COROLLARY 4.4.4. If each $A \in A$ is $G X$ for some $X \in S$ then $S_{G}$ is dense and codense in PC.

Most of these results are valid for $V=A b$; however, for general $V$, one must postulate that $E-M$ is suitably related to $C$ in the first place.

EXAMPLE 4.5. If a composite functor of the form

$$
\mathrm{K} \stackrel{J}{\longrightarrow} \mathrm{A} \stackrel{N}{\longrightarrow} \mathrm{C}
$$

is dense then we can ask when the resultant expression $C(N J k, C) \circ N J k \cong C$ gives a density presentation of $N$. Clearly this is so iff

$$
C(N J k, C) \circ \mathrm{A}(A, \pi k) \cong \mathcal{C}(N A, C)
$$

for all $A \in A$ and $C \in \mathcal{C}$. If $N$ is a full embedding this is so iff $J$ is Cauchy dense in the sense that

$$
\int^{k} A\left(J k, A^{\prime}\right) \otimes A(A, J k) \cong A\left(A, A^{\prime}\right)
$$

for all $A, A^{\prime} \in A$.

For example, let $V=R^{+}$with the usual monoidal closed structure generated by addition on $\mathrm{R}^{+}$(Lawvere [18]). Then $V$-categories are 
simply metric spaces in a suitably general sense. The unit circle is $V$-dense in the unit disc in $C$, a presentation being given by the rational points on the circle. The Kan extension (Day [8]) of addition of the rational points is the multiplication of the points in the disc.

EXAMPLE 4.6. Suppose that $N: A \rightarrow C$ is a functor and, for each $C \in|C|$, there exists a free V-category $K_{C}$ and a functor $P_{C}: K_{C} \rightarrow A$ together with a colimit cone $\xi_{C}: \operatorname{colim}_{k} N P k \cong C$ such that $\operatorname{colim}_{k} A(A, P K) \cong \mathcal{C}(N A, C)$ for all $A \in A$. Then $(P, K, \xi)$ determines a density presentation of $\mathcal{C}$ on setting

$$
K=\sum_{|\bar{C}|} K_{C} \text { and } J=\left(P_{C}\right): K \rightarrow A \text {, }
$$

and defining $K: K^{\mathrm{OP}} \otimes|\mathrm{C}| \rightarrow V$ by the Kronecker condition

$$
\left\{\begin{array}{l}
K(k, C)=I \text { if } k \in K_{C}, \\
K(k, C)=0 \text { if } k \notin k_{C} .
\end{array}\right.
$$

For example, if $v=S$ then the "discrete" comma-category presentation of a dense functor $N$ is given by $K_{C}=N / C$ for each $C \in \mathcal{C}$. Furthermore, given any functor $N: A \rightarrow C$ the condition $\operatorname{colim}_{k} A(A, P k) \cong C(N A, C)$ is satisfied for all $A \in A$ and $C \in C$ by the representation theorem. Thus, by Proposition 1.3, we have:

PROPOSITION 4.6.1. $N: A \rightarrow C$ is dense iff $N$ is strongly generating and $C\left(N A, \operatorname{colim}_{k} N P k\right) \cong \operatorname{colim}_{k} A(A, P k)$ for all $A \in A$ and $C \in \mathcal{C}$

In particular, if $C$ is an $S$-based category and $\alpha$ is a regular cardinal number then $A \in C$ is called a-presentable if $\mathcal{C}(A,-): \mathcal{C} \rightarrow S$ preserves $\alpha$-filtered colimits (see Gabriel, Ulmer [13]). Let $C_{\alpha}$ denote the full subcategory of $\mathcal{C}$ determined by the $\alpha$-presentable objects.

COROLLARY 4.6.2 (Gabriel, Ulmer [13], Theorem 7.4). A composite inclusion of the form $A \subset C_{\alpha} \subset C$ is dense if $A$ strongly generates $C$ and $A / C$ is a-filtered for each $C \in C$; in particular, if $A$ is closed 
under a-filtered colimits in $C . \quad / /$

A cocomplete category $C$ is then said to be locally $\alpha$-presentable if it contains a small strongly generating set of $\alpha$-presentable objects. For results involving the characterisation of locally $\alpha$-presentable categories we refer directly to Diers [10], Example 5.3.

Again suppose that $V=S$. An object $C \in C$ is a Z-object (is "connected") if the functor $\mathcal{C}(C,-): \mathcal{C} \rightarrow S$ preserves coproducts in $\mathcal{C}$. An object $C \in C$ is $z$-generated if $C=\sum C_{i}$ with each $C_{i}$ a z-object.

PROPOSITION 4.6.3 (R.-E. Hoffmann). The inclusion of the category of Z-objects in the category of Z-generated objects is dense. I/

EXAMPLE 4.7. Suppose $\xi_{C}: K_{C}(k) \circ N J C^{k} \cong C$ is given for each $C \in C$ where $K_{C}: K_{C}^{\mathrm{op}} \rightarrow v$ and $J_{C}: K_{C} \rightarrow A$ and $K_{C}(k) \circ \mathrm{A}\left(A, J_{C} k\right) \cong \mathcal{C}(N A, C)$ for all $A \in A$ and $C \in \mathcal{C}$. Then a presentation of $N: A \rightarrow C$ is given by

$$
K=\sum_{C \in T C \mid} K_{C} \text { and } J=\left(J_{C}\right): K \rightarrow A
$$

with

$$
\begin{cases}K(k, C)=K_{C}(k) & \text { if } k \in K_{C}, \\ K(k, C)=0 & \text { if } k \notin K_{C} .\end{cases}
$$

For example, if $V=S$ and $N: A \rightarrow C$ is dense then $N_{B}: N / B \rightarrow C / B$ is dense for all $B \in C$ because $N / C \cong N_{B} / g$ for all $g \in C(C, B)$. A presentation of $N_{B}$ is given on setting $K_{g}=N / C$ and $J_{g}=N / g: N / C \rightarrow N / B$ for each $g \in \mathcal{C}(C, B)$ and $C \in \mathcal{C}$. The coefficients are then given by $K_{g}: N / C^{\mathrm{Op}} \rightarrow S, K_{g}(x)=C(N A, C)$ where $x: N A \rightarrow C$. The result is the "reduced" coend presentation of $N_{B}$ : 


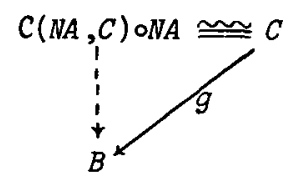

This example extends to any cartesican closed category $V$.

\section{Linear monads}

Many of the density presentations arising in monad theory are not strict. Thus a more complex form of Theorem 3.1 is often needed. Here, however, we are mainly concerned with the presentations themselves and results along the lines of Theorem 3.1 will appear elsewhere.

Suppose that a monad $T=\left(T, \mu_{3} \eta\right)$ is given on $C$ and denote the usual resolution of this monad into a Kleisli category and an EilenbergMoore category by

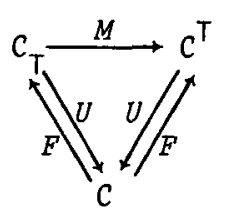

where $M$ is the dense comparison functor.

PROPOSITION 5.1. Given a dense functor $N: A \rightarrow \mathrm{C}_{\mathrm{T}}$ the composite MN : $\mathrm{A} \rightarrow \mathrm{C}^{\top}$ is dense.

Proof. Because $N$ is dense we have $C \cong C_{\top}(N A, C) \circ N A$ in $C_{\mathrm{T}}$. Thus $M C \cong C_{T}(N A, C) \circ M N A$ for each $\left(C, \zeta_{C}\right) \in C^{\top}$. Thus there exists a density presentation of $M N$ with structure $\zeta_{C}: M C \rightarrow C \cdot / /$

The concept of a density presentation is related to the idea of a moned with rank (see Barr [2] and [3], Freyd and Kelly [12], Gabriel, Ulmer [13], and Linton [20]). Suppose $N: A \rightarrow C$ is a full embedding with density presentation $P=(J, K, \xi)$.

DEFINITION 5.2. (a) $T$ is a P-monad if $C(N A, T \xi)$ and $K(k, C) \circ \mathcal{C}(N A, T N J k) \rightarrow \mathcal{C}(N A, T(K(k, C) \circ N J k))$ are both epimorphisms for all $A \in A$ and $C \in C$. 
(b) $\mathrm{T}$ is P-linear if $K(k, C)$ o TNJk exists in $C$ for all $C \in \mathcal{C}$ and the canonical maps $K(k, C) \circ T N J k \rightarrow T C$ are epimorphisms for all $c \in C$.

PROPOSITION 5.3. A P-monad is P-Zinear.

Proof . Consider the diagram

$$
\int^{k} K(k, C) \otimes \mathcal{C}(N A, T N J k) \rightarrow \mathcal{C}(N A, T(K(k, C) \circ N J k))
$$

The diagonal is an epimorphism because $T$ is a P-monad. Thus, by density of $N$, the maps $K(k, C) \circ T N J k \rightarrow T C$ are epimorphisms. //

For the given density presentation $P$ of $N$ define categories $K^{\prime}$ and $A^{\prime}$ and functors $J^{\prime}$ and $N^{\prime}$ as follows: $A^{\prime}$ is the full image of $F N: A \rightarrow C_{T}, K^{\prime}$ is the full image of $F_{J}: K \rightarrow A^{\prime}$, and $N_{-}^{\prime}: A^{\prime} \rightarrow C_{T}$, $J^{\prime}: K^{\prime} \rightarrow A^{\prime}$ are the induced functors such that $F N=N^{\prime} F$ and $F J=J^{\prime} F$ :

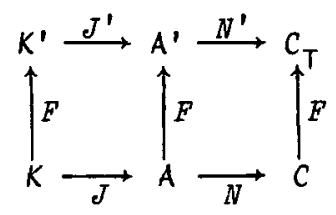

A coefficient functor $K^{\prime}:\left(K^{\prime}\right)^{\mathrm{OP}} \times\left|\mathrm{C}_{T}\right| \rightarrow V$ is defined as $K^{\prime}(F h, F C)=K^{\prime}(F h, F k) \circ K(k, C)$, and $\xi^{\prime}: K^{\prime}(F h, F C) \circ N^{\prime} J^{\prime}(F h)+F C$ is defined by:

$K^{\prime}(F h, F C) \circ N^{\prime} J^{\prime}(F h)=\left(K^{\prime}(F h, F k) \circ K(k, C)\right) \circ N^{\prime} J^{\prime}(F h)$ $\cong K(k, C) \circ N^{\prime} J^{\prime}(F k)$ by the representation theorem $\cong k(k, c) \circ$ FNJk $\cong F(K(k, C) \circ N J k)$ $\rightarrow F C$,

thus giving a presentation $P^{\prime}$ of $N^{\prime}$.

THEOREM 5.4. $T$ is a $P_{\text {-monad iff }} P^{\prime}$ is a density presentation of $N^{\prime}$.

Proof. Because $F \dashv U, C_{T}\left(N^{\prime} F A, \xi_{F C}^{\prime}\right)$ is an epimorphism for all 
$F A \in A^{\prime}$ iff $\mathrm{C}(N A, T \xi)$ is an epimorphism for all $A \in A$;

$$
\mathcal{C}\left(N^{\prime} F A, \xi_{F}^{\prime}\right) \cong \mathcal{C}\left(F N A, \xi_{F C}^{\prime}\right) \cong C\left(N A, T \xi_{C}\right) \text {. }
$$

Moreover,

$$
\begin{aligned}
K^{\prime}(F k, F C) \circ A^{\prime}\left(F A, J^{\prime} F k\right) & =\left(K^{\prime}(F k, F h) \circ K(h, C)\right) \circ A^{\prime}\left(F A, J^{\prime} F k\right) \\
& \cong K(h, C) \circ A^{\prime}\left(F A, J^{\prime} F h\right) \\
& \cong K(h, C) \circ C_{T}\left(N^{\prime} F A, N^{\prime} J^{\prime} F h\right) \\
& \cong K(h, C) \circ C^{\top}(F N A, F N J h) \\
& \cong K(h, C) \circ C(N A, T N J h) \\
& \rightarrow C(N A, T(K(k, C) \circ N J k)) \\
& \cong C(N A, U(K(k, C) \circ F N J k)) \text { since } F \rightarrow U \\
& \cong C_{T}\left(F N A, K^{\prime}(F k, F C) \circ N^{\prime} J^{\prime} F k\right)
\end{aligned}
$$

which is an epimorphism iff

$$
K(h, C) \circ(N A, T N J h)+C(N A, T(K(h, C) \circ N J h))
$$

is an epimorphism. $/ /$

In order to demonstrate the relationship of $P$-monads to monads with rank consider Proposition 4.6.2 where $V=S$ and $\alpha$ is a regular cardinal. Suppose each $A / C$ is $\alpha$-filtered and $P_{C}: A / C \rightarrow A$ be projection. Suppose $A$ is small and $C$ is cocomplete.

PROPOSITION 5.5. The following conditions are equivanlent:

(a) $\int^{B} \mathcal{C}(N B, C) \times \mathcal{C}(N A, T N B) \cong \mathcal{C}(N A, T C)$;

(b) $\int^{A} \mathcal{C}(N A, C) \cdot T N A \cong T C$;

(c) $T$ preserves colim $N P C^{k}$ for all $C \in \mathcal{C}$;

(d) $T$ preserves $\alpha$-filtered colimits.

Proof. (b) $\Rightarrow$ (d) since $N A \in C_{\alpha}$ for all $A \in A$. (d) $\Rightarrow$ (c) because $\mathrm{A} / C$ is $\alpha$-filtered for all $C \in \mathcal{C} .($ (c) $\Rightarrow$ (b) because 


$$
\begin{aligned}
& \int^{A} C(N A, C) \cdot T N A \cong \int^{A} \mathcal{C}\left(N A, \operatorname{colim} N P C^{k}\right) \cdot T N A \\
& \cong \operatorname{colim} \int^{A} \mathrm{c}\left(N A, N P C^{k}\right) \cdot T N A \text { because } N A \in C_{\alpha} \\
& \cong \operatorname{colim} T N P C^{k} \text { by the representation theorem } \\
& \cong T\left(\operatorname{colim} N P C^{k}\right) \text { by }(c) \\
& \cong T C \text {. } \\
& \int^{B} \mathrm{C}(N B, C) \times C(N A, T N B) \cong \int^{B} \mathrm{C}\left(N B, \operatorname{colim} N P C^{k}\right) \times C(N A, T N B) \\
& \cong \operatorname{colim} \int^{B} \mathrm{C}\left(N B, N P C^{k}\right) \times C(N A, T N B) \\
& \cong \operatorname{colim} C\left(N A, T N P C^{k}\right) \\
& \text { by the representation theorem } \\
& \cong \mathcal{C}\left(N A \text {, colim } T N P C_{C}\right) \text { because } N A \in \mathcal{C}_{\alpha} \\
& \cong \mathcal{C}\left(N A, T\left(\operatorname{colim} N P C^{k}\right)\right) \cong \mathcal{C}(N A, T C) \text {. } / /
\end{aligned}
$$

Suppose that $A_{0} \in A$ is a base object such that $N A \cong \mathcal{C}\left(N A_{0}, N A\right) \cdot N A_{0}$ and let $P$ be the standard coend presentation of $N: \mathrm{A} \rightarrow \mathrm{C}$.

PROPOSITION 5.6. $T$ is a P-monad iff

$$
\int^{B} \mathrm{C}(N B, C) \times \mathcal{C}\left(N A_{0}, T N B\right) \rightarrow \mathcal{C}\left(N A_{0}, T C\right)
$$

is an epimorphism.

Proof. Necessity is clear. For sufficiency, we have 


$$
\begin{aligned}
& \int^{B} \mathrm{C}(N B, C) \times \mathrm{c}(N A, T N B) \cong \int^{B} \mathrm{c}\left(N B, \operatorname{colim} N P C^{k}\right) \times c(N A, T N B) \\
& \cong \operatorname{colim} C\left(N A, T N P C^{k}\right) \\
& \cong C\left(N A, \operatorname{colim} T N P C^{k}\right) \\
& \cong \simeq\left[\mathrm{C}\left(N A_{0}, N A\right), C\left(N A_{0}, \operatorname{colim} T N P C^{k}\right)\right] \\
& \cong\left[\mathrm{C}\left(N A_{0}, N A\right), \int^{B} \mathrm{C}(N B, C) \times \mathrm{C}\left(N A_{0}, T N B\right)\right] \\
& \rightarrow\left[C\left(N A_{0}, N A\right), C\left(N A_{0}, T C\right)\right] \\
& \text { because epimorphisms split in } S \\
& \cong \simeq C(N A, T C) \text {. // }
\end{aligned}
$$

Thus, if we regard $\mathrm{C}\left(N A_{0}, T C\right)$ as the set of $C$-ary operations of $T$ then $T$ is a $P$-monad iff each $C$-ary operation factors appropriately through some $N A$-ary operation. With suitable modifications $N A_{0}$ can clearly be replaced by a dense subcategory of $C$ which lies in $A$.

Instances of $P$-monads occur widely in finitary universal algebra over a suitable closed category $V$ (see Borceux and Day [4]).

\section{References}

[1] H. Appelgate and M. Tierney, "Categories with models", Seminar on triples and categorical homology theory, 156-244 (Lecture Notes in Mathematics, 80. Springer-Verlag, Berlin, Heidelberg, New York, 1969).

[2] Michael Barr, "Coequalisers and free triples", Math. Z. 116 (1970), 307-322.

[3] Michael Barr, "Exact categories", Exact categories and categories of sheaves, 1-120 (Lecture Notes in Mathematics, 236. SpringerVerlag, Berlin, Heidelberg, New York, 1971).

[4] Francis Borceux and Brian Day, "Universal algebra in a closed category", j. Pure Appl. Algebra (to appear).

[5] Francis Borceux and G.M. Kelly, "A notion of limit for enriched categories", Bull. Austral. Math. Soc. 12 (1975), 49-72. 
[6] Marta C. Bunge, "Relative functor categories and categories of algebras", J. Algebra 11 (1969), 64-101.

[7] Brian Day, "On closed categories of functors II", Category seminar, 20-54 (Proc. Sydney Category Seminar 1972/1973. Lecture Notes in Mathematics, 420. Springer-Verlag, Berlin, Heidelberg, New York, 1974).

[8] B.J. Day, "Varieties of a closed category", BulZ. Austral. Math. Soc. 16 (1977), 131-145.

[9] Yves Diers, " $J$-adjonction, limite $J$-absolue et $J$-théorie algêbrique", C.R. Acad. Sci. Paris Sér. A 278 (1974), 1009-1012.

[10] Yves Diers, "Type de densité d'une sous-catégorie pleine", Ann. Soc. Sci. Bruxelles Sér. I 90 (1976), 25-47.

[11] Samuel Eilenberg and G. Max Kelly, "Closed categories", Proc. Conf. Categorical Algebra, La Jolla, California, 1965, 421-562 (Springer-Verlag, Berlin, Heidelberg, New York, 1966).

[12] P.J. Freyd and G.M. Kelly, "Categories of continuous functors, I", J. Pure Appl. Algebra 2 (1972), 169-191.

[13] Peter Gabriel, Friedrich UImer, Lokal präsentierbare Kategorien (Lecture Notes in Mathematics, 221. Springer-Verlag, Berlin, Heidelberg, New York, 1971).

[14] Dion Gildenhuys and Chong-Keang Lim, "Free pro-C-Groups", Math. 2. 125 (1972), 233-254.

[15] Karl Heinrich Hofmann, "Category theoretical methods in topological algebra", Categorical topology, 345-403 (Proc. Conf. Mannheim, 1975. Lecture Notes in Mathematics, 540. Springer-Verlag, Berlin, Heidelberg, New York, 1976).

[16] J.R. Isbell, "Adequate subcategories", IZZinois J. Math. 4 (1960), 541-552.

[17] J.F. Kennison and Dion Gildenhuys, "Equational completions, model induced triples and pro-objects", J. Pure Appl. Algebra 1 (1971), 317-346. 
[18] F. William Lawvere, "Metric spaces, generalized logic, and closed categories", Lecture Notes, Istituto di Matematica, Università di Perugia.

[19] Lim Chong-Keang, "Tripleableness of pro-C-groups", J. Austral. Math. Soc. Ser. A 21 (1976), 299-309.

[20] F.E.J. Linton, "Some aspects of equational categories", Proc. Conf. Categorical Algebra, La Jolla, California, 1965, 84-94 (Springer-Verlag, Berlin, Heidelberg, New York, 1966).

[21] S. Mac Lane, Categories for the working mathematicion (Graduate Texts in Mathematics, 5. Springer-Verlag, New York, Heidelberg, Berlin, 1971).

[22] Friedrich UImer, "Properties of dense and relative adjoint functors", J. Azgebra 8 (1968), 77-95.

Department of Pure Mathematics,

University of Sydney,

Sydney,

New South Wales. 